\title{
Impactos financeiros da pandemia da COVID-19 na aquisição de medicamentos do componente básico em um município do interior da Bahia-Brasil
}

\author{
Financial impacts of the COVID-19 pandemic on the acquisition of basic component drug in a \\ municipality in the interior of Bahia-Brazil
}

Impactos financieros de la pandemia COVID-19 en la adquisición de fármaco componente básico en un municipio del interior de Bahia-Brasil

Recebido: 21/11/2021 | Revisado: 29/11/2021 | Aceito: 01/12/2021 | Publicado: 12/12/2021

\author{
Gleiciane Pateis Matos \\ ORCID: https://orcid.org/0000-0003-0019-9374 \\ Faculdade Independente do Nordeste, Brasil \\ E-mail: gleicy03pateis@gmail.com \\ Josiene Macedo Neres \\ ORCID: https://orcid.org/0000-0001-9706-4876 \\ Faculdade Independente do Nordeste, Brasil \\ E-mail: josimneres@outlook.com \\ Tayanne Andrade dos Santos \\ ORCID: https://orcid.org/0000-0002-7977-6308 \\ Faculdade Independente do Nordeste, Brasil \\ E-mail:tayanne.as@hotmail.com
}

\begin{abstract}
Resumo
A pandemia causada pelo novo coronavírus, SARS-CoV-2, estabeleceu uma realidade alarmante a toda organização que se refere a saúde. O setor farmacêutico foi um dos afetados, sofrendo impacto direto em decorrência do desabastecimento de medicamentos, insumos e correlatos. O custo impremeditável e a inflação exacerbada aos itens de saúde, ultrapassaram todos os outros setores da economia, impactando diretamente nos usuários do sistema único de saúde (SUS). Dessa maneira o objetivo da pesquisa foi identificar e analisar os impactos da pandemia da COVID19 na aquisição de medicamentos do componente básico do município de Vitória da Conquista-Bahia. As amostras utilizadas no estudo foram coletadas através do diário oficial do município por meio das atas de registro de preço dos medicamentos listados na relação de medicamentos essenciais do município (REMUME) nos anos de 2018, 2019 e 2020. A investigação caracterizou-se por ser uma pesquisa documental, de abordagem quali-quantitativa. Foram analisados dados de 96 medicamentos no total, visto que, os anti-hipertensivos foram os que se mantiveram constante, ou seja, não teve diminuição em sua quantidade licitada, tendo em vista que alguns medicamentos fracassaram devido a urgência e demanda elevada havendo a necessidade de um parecer jurídico para que eles fossem comprados. A pandemia da COVID-19 impactou negativamente na aquisição de medicamentos do município de Vitória da Conquista, fazendo com que cerca de 11 medicamentos acima de 30.000 itens sofressem reequilíbrio-econômicofinanceiro e tivesse muitas licitações fracassadas levando a escassez de alguns medicamentos por meses, tendo assim a necessidade de capacitar os gestores para melhor articulações frente ao mercado global.
\end{abstract}

Palavras-chave: Pandemia; Medicamentos; Assistência farmacêutica.

\begin{abstract}
The pandemic caused by the new coronavirus, SARS-CoV-2, has established an alarming reality for every organization concerned with health. The pharmaceutical sector was one of the most affected, suffering direct impact due to the lack of supply of medicines, supplies and related products. The unpredictable cost and exacerbated inflation to health items have overtaken all other sectors of the economy, impacting directly the users of the Brazilian Health System (SUS). Thus, the objective of this research was to identify and analyze the impacts of the COVID-19 pandemic on the purchase of medicines of the basic component in the municipality of Vitória da Conquista-Bahia. The samples used in the study were collected through the official register of the municipality by means of the bidding protocol of the medicines listed in the Municipal Essential Medicines Lists (REMUME) in the years 2018, 2019 and 2020. The investigation was characterized as a documentary research, with a qualitative-quantitative approach. A total of 96 medicines were analyzed, since the anti-hypertensive drugs were the ones that remained constant, in other words, there was no decrease in the quantity bid for, bearing in mind that some medicines failed due to urgency and high demand, requiring a legal opinion for them to be purchased. The COVID-19 pandemic has negatively impacted the purchase of medicines in the municipality of Vitória da Conquista, causing around 11 medications above 30,000
\end{abstract}


items to undergo economic-financial rebalancing and having many failed bids leading to a shortage of some medications for months, thus having the need to train managers to better articulate with the global market.

Keywords: Pandemic; Medicines; Pharmaceutical assistance.

\begin{abstract}
Resumen
La pandemia causada por el nuevo coronavirus, SARS-CoV-2, ha establecido una realidad alarmante para todas las organizaciones relacionadas con la salud. El sector farmacéutico fue uno de los afectados, sufriendo un impacto directo como consecuencia de la escasez de medicamentos, insumos y productos relacionados. El costo impredecible y la inflación exacerbada de los artículos de salud, superó a todos los demás sectores de la economía, impactando directamente a los usuarios del Sistema Único de Salud (SUS). Así, el objetivo de la investigación fue identificar y analizar los impactos de la pandemia COVID-19 en la adquisición de medicamentos del componente básico en la ciudad de Vitória da Conquista-Bahia. Las muestras utilizadas en el estudio fueron recolectadas a través del diario oficial de la ciudad a través de los registros de precio de los medicamentos que figuran en el listado de medicamentos esenciales de la ciudad (REMUME) en los años 2018, 2019 y 2020. La investigación se caracterizó por ser un investigación documental, con enfoque cuali-cuantitativo. Se analizaron datos de 96 medicamentos en total, ya que los antihipertensivos fueron los que se mantuvieron constantes, es decir, no hubo disminución en la cantidad de su oferta, considerando que algunos medicamentos fallaron por urgencia y alta demanda. comprado. La pandemia de COVID19 afectó negativamente la compra de medicamentos en la ciudad de Vitória de Conquista, provocando que alrededor de 11 medicamentos por encima de 30.000 ítems sufran un reequilibrio económico-financiero y que muchas licitaciones fallidas provoquen una escasez de algunos medicamentos durante meses, teniendo así la necesidad de capacitar a los gerentes para una mejor articulación con el mercado global.
\end{abstract}

Palabras clave: Pandemia.; Medicamentos; Cuidado farmacéutico.

\title{
1. Introdução
}

A COVID-19, designação dada pela organização mundial de saúde (OMS), é uma doença causada pelo novo coronavírus da síndrome respiratória grave, o vírus SARS-CoV-2, identificada pela primeira vez em Wuhan, na China em dezembro de 2019 O vírus apresenta um espectro clínico que varia de casos assintomáticos a quadros graves, possuindo sintomatologia ampla e diagnóstico complexo. A transmissão ocorre a partir de gotículas de saliva através do contato com outros indivíduos contaminados (Farinha, 2020; Silva, 2020).

Quase dois anos após o início da pandemia, no Brasil uma crise sanitária é presenciada. Vários fatores contribuem para essa circunstância, como o processo tardio de imunização coletiva, escassez de EPIs e desabastecimento de medicamentos tanto para o tratamento do SARS-CoV-2, como na atenção básica e especializada disponibilizada pelo sistema único de saúde. A dificuldade na aquisição de medicamentos pode estar relacionada as medidas preventivas mundiais que impossibilitaram a extração de matéria prima afetando tanto a produção quanto a importação desses produtos. Outro aspecto responsável pelas objeções de compra, foi a redução de investimentos federais nas indústrias farmacêuticas brasileiras, o que culminou na falta de manufatura dos fármacos e a divergência logística de importação, em razão do transporte realizado ser majoritariamente aéreo, método esse que sofreu redução devido a pandemia mundial (Almeida, 2020; Alves, 2020; Junqueira, 2020).

Os inesperados gastos da pandemia e a própria inflação direcionada aos itens de saúde ultrapassou todos os outros setores da economia, havendo assim uma contribuição para que muitos medicamentos oscilassem de preços tão repentinamente que o setor público não conseguisse efetuar a compra. Setor esse, denominado assistência farmacêutica, responsável por gerenciar, planejar e organizar atividades que visam a otimização dos serviços oferecidos à população, com propósito de desenvolver e ampliar estratégias para o acesso igualitário aos medicamentos considerados indispensáveis, sendo definitivo para a resolubilidade da atenção e dos serviços em saúde que envolvem a alocação de verbas. A implementação da assistência é desenvolvida com o auxílio de uma esquematização constituída pelas etapas de seleção, programação, aquisição, armazenamento, distribuição e dispensação (Feliciano, Bezerra \& Santos, 2019; Menezes, Moretti \& Reis, 2019).

Na gestão farmacêutica, estes gastos são expressivos, em função do alto valor unitário dos medicamentos ou pelo grande volume de itens necessários para suprir as necessidades da população (Brasil, 2007). O financiamento da assistência farmacêutica é descentralizado e os recursos advindos do governo Federal e Estadual são repassados principalmente pelo 
gerenciamento fundo a fundo, que são depósitos de verbas federais realizados por meio de transferência do Fundo Nacional de Saúde para os Fundos de Saúde estaduais e municipais. Segundo a Portaria No 3992/2017, a Assistência farmacêutica está inserida no bloco de custeio, sendo fragmentada em componentes, estratégico, especializado e básico, sendo o recurso repassado obedecendo a especificidade de cada grupo (Cerqueira, 2018; RENAME, 2001; Brasil, 2017).

O componente básico (CBAF) refere-se à aquisição de medicamentos e insumos da atenção básica em saúde e àquelas relacionadas a agravos e programas de saúde específicos. Segundo a Portaria n ${ }^{\circ}$ 3.193/2019 que regulariza o financiamento do CBAF, dispõe que a alocação de verbas para o repasse, serão definidos com base no Índice de Desenvolvimento Humano Municipal (IDHM), considerando o número de habitantes (per capita/ano) de cada município e repassando o valor de referência do ente federativo por habitante. $\mathrm{O}$ repasse é realizado em 12 parcelas, utilizando a população estimada pelo Instituto Brasileiro de Geografia e Estatística (IBGE) como referência. Esse recurso é destinado à aquisição dos medicamentos e insumos constantes nas relações nacionais (RENAME), estaduais (RESME) e municipais (REMUME) de medicamentos essenciais. (Brasil, 2019; Beltrane, Pontarolli, Cortez \& Rieck, 2007).

Conforme o IBGE, a população estimada em Vitória da conquista no ano de 2021 contabilizou 343.643 habitantes, sendo o seu último IDHM calculado em 2010, com base na população do mesmo ano que estava em torno de 306.866 habitantes, gerando um IDHM de 0,678. Segundo a PORTARIA No 3.193/2019, o município possui IDHM médio, recebendo 5,95 R \$ por habitante/ano para aquisição de medicamentos para o componente básico. A modalidade de compra utilizada é o pregão eletrônico, destinado à compra de qualquer valor, de produtos de bem comum. Quando há fracasso em licitações ou quando é necessário efetuar a aquisição por urgência, há uma modalidade denominada dispensa de licitação, cuja justificativa permite a compra sem estabelecer uma modalidade de compra vigente (IBGE, 2021; Lôbo, 2019; Brasil, 2019).

No município, a modalidade de licitação é tipificada em atas de registro de preço. Nesse sistema de contratação, o órgão deve possuir uma previsão da quantidade que irá precisar durante o ano e que será comprada, assim realizará o edital embasado nessa estimativa. A empresa que ganhar a licitação assina um termo de contrato se responsabilizando manter o preço ofertado por todo o período vigente da ata, além de ter que cumprir todas as cláusulas impostas no edital. Vale ressaltar que as atas de registro de preço, são apenas uma previsão, pois o contratante não é obrigado a comprar toda a quantidade estimada e nem de uma só vez, podendo realizar a aquisição de maneira fracionada, na quantidade que for necessária, visto que, na ata está estabelecido o valor unitário do produto ou serviço., podendo ser requisitado a qualquer momento (Bermudez, 2017).

Tanto o art. $\mathrm{n}^{\circ} 65$ da Lei 8.666/93, quanto o decreto 7.892/13 regulamenta que os preços registrados nas atas podem ser revistos em decorrência da variação do mercado e de fatores que podem alterar o preço registrado. Nesse caso o valor da Ata poderá ser recomposto mediante o pedido de "reequilíbrio econômico-financeiro". Essa solicitação pode ser feita a administração, visto que os fornecedores possuam documentos comprobatórios da defasagem do preço. O decreto 7.892/13 regulamenta também o cancelamento da ata devido a mesma situação de defasagem dos valores em relação ao mercado, porém deverá ser feito mediante requerimento, com a documentação comprobatória da impossibilidade de manter o preço e de cumprir o fornecimento. (Brasil, 1993; Brasil, 2013).

Desse modo, o objetivo dessa pesquisa foi avaliar os impactos financeiros da pandemia da COVID-19 na aquisição de medicamentos essenciais para o município de Vitória da Conquista, Bahia, visando determinar os medicamentos listados na RENAME/REMUME que obtiveram aumento de preço nos anos de 2018, 2019 e 2020.

\section{Metodologia}

Trata-se de uma pesquisa de caráter documental, com abordagem qualitativa e quantitativa. A pesquisa documental é similar a bibliográfica, diferenciando apenas entre a natureza das fontes, visto que a bibliográfica busca fundamentos de diversos autores, enquanto, a documental utiliza-se materiais que ainda não receberam um tratamento analítico. Já a 
perspectiva qualitativa tem como objetivo compreender o que se estuda, ou seja, busca a especificação das variedades de representações das pessoas no seu mundo vivencial. Enquanto a abordagem quantitativa, é baseado em ideias pré-concebidas de modo que os conceitos estão relacionados sendo compostas por variáveis quantificadas em números, que são analisadas de modo estatístico com o propósito de estipular se os dados previstos na teoria se sustentam ou não (Gil, 1999; Silva, 2018).

A pesquisa foi realizada em Vitória da Conquista, Bahia, Brasil, a partir das atas de registro de preço dos medicamentos listados na REMUME nos anos de 2018, 2019 e 2020. Esses documentos estão disponíveis no diário oficial do município. Essa ferramenta é disponibilizada para que se torne pública toda a atividade administrativa municipal, como consta no artigo $\mathrm{N}^{\circ} 37$ da constituição federal que dispõe sobre os princípios organizacionais do Estado, dentre eles a publicidade dos atos. Integrado as atas, foram analisados também todos os dados que possuem reequilíbrios financeiros dos respectivos registros e quantitativos licitados. As atas de registro de preço, que tem por objeto protocolar e formalizar valores para aquisições pela modalidade de compra licitatória. Nessas atas direcionadas a compra de medicamentos geralmente constam os valores unitários de cada item, o quantitativo a ser licitado e o valor total da licitação.

O procedimento consistiu em busca ativa no diário oficial do município pelas atas de registro de preço dos medicamentos nos anos de 2018, 2019 e 2020 utilizando as palavras-chave, "atas de registro de preço", "medicamentos", "assistência farmacêutica". Foram excluídas todas as atas que não se enquadravam nesses anos, e todos os medicamentos e insumos que não pertenciam a REMUME. Selecionamos para estudo apenas os medicamentos que tiveram licitação acima de 30.000 itens. Posteriormente avaliamos as licitações e seus valores unitários comparados dentre os anos, para demonstrar o quanto a pandemia impactou na aquisição desses medicamentos citados.

\section{Resultados e Discussão}

Foram analisados dados de 96 medicamentos no total. Em 2018, 2019 e 2020, segundo a Tabela 1, alguns medicamentos foram comprados em uma quantidade inferior a 30.000, impedindo o comparativo com outros anos determinados, mas que houve a aquisição.

Nos anos selecionados o número de licitações que lograram êxito sofreu variações. Considerando o quantitativo estabelecido acima de 30.000 unidades, no ano de 2018, foram licitados 86 medicamentos, em 2019, 84 obtiveram êxito e no ano de 2020, seguindo os critérios para análise, apenas 78 foram comprados. Essa queda no número de licitações realizadas no último ano citado, tem relação direta com a pandemia, visto que, os empenhos foram executados no primeiro semestre do ano, momento no qual o número de casos de COVID-19 aumentava exorbitantemente sendo um ensejo para a OMS declarar a existência de uma pandemia mundial, descartando a premissa de epidemia ou surtos isolados. No ano de 2020, como denota a Tabela 2 foi o ano analisado que obteve mais fracassos em licitação totalizando 15 medicamentos, 60\% do número de fracassos nos anos analisados. Os medicamentos que fracassaram no pregão não estão ligados diretamente com o desabastecimento no serviço, uma vez que os fármacos podem ser adquiridos mediante dispensa de licitação, que consiste em uma aquisição sem estabelecer uma modalidade vigente, cuja justificativa para esse ato deva ser plausível. 
Tabela 1: Medicamentos da atenção básica que tiveram licitação acima de 30 mil unidades.

\begin{tabular}{|c|c|c|c|}
\hline MEDICAMENTOS & $\begin{array}{l}\text { QUANT. LICITADA } \\
2018\end{array}$ & $\begin{array}{l}\text { QUANT. LICITADA } \\
2019\end{array}$ & $\begin{array}{l}\text { QUANT. LICITADA } \\
2020\end{array}$ \\
\hline \multicolumn{4}{|c|}{ ANTI-HIPERTENSIVOS } \\
\hline Anlodipino 5mg comprimido & 2.000 .000 & 2.000 .000 & 2.000 .000 \\
\hline Atenolol 50mg comprimido & 1.500 .000 & 1.500 .000 & 1.500 .000 \\
\hline $\begin{array}{l}\text { Enalapril, } \\
\text { comprimido. }\end{array}$ & 4.000 .000 & 4.000 .000 & 4.000 .000 \\
\hline Enalapril, maleato 5mg comprimido. & 1.500 .000 & 1.500 .000 & 1.500 .000 \\
\hline $\begin{array}{l}\text { Metildopa 250mg comprimido em } \\
\text { blister }\end{array}$ & 500.000 & 500.000 & 500.000 \\
\hline Propanolol 40mg comprimido & 1.000 .000 & 1.000 .000 & 1.000 .000 \\
\hline Verapamil 120 mg comprimido & 100.000 & 100.000 & 100.000 \\
\hline Verapamil $80 \mathrm{mg}$ comprimido & 100.000 & 100.000 & 100.000 \\
\hline Espironolactona $25 \mathrm{mg}$ comprimido & 400.000 & 400.000 & 400.000 \\
\hline Furosemida 40mg comprimido & 600.000 & 1.500 .000 & 1.500 .000 \\
\hline Carvediol 12,5 mg comprimido & 500.000 & 500.000 & 500.000 \\
\hline Carvediol 3,125 mg comprimido & 500.000 & 500.000 & 500.000 \\
\hline \multicolumn{4}{|c|}{ HIPOGLICEMIANTES/ANTIDIABÉTICOS } \\
\hline $\begin{array}{l}\text { Gliclazida 30mg comprimido de } \\
\text { liberação controlada }\end{array}$ & 1.000 .000 & 250.000 & 250.000 \\
\hline \multicolumn{4}{|c|}{ ANTILIPIDÊMICO } \\
\hline Sinvastatina $20 \mathrm{mg}$ comprimido & 2.500 .000 & 2.500 .000 & 2.500 .000 \\
\hline \multicolumn{4}{|c|}{ ANTIANGINOSOS } \\
\hline Digoxina $0,25 \mathrm{mg}$ comprimido & 200.000 & 200.000 & 200.000 \\
\hline 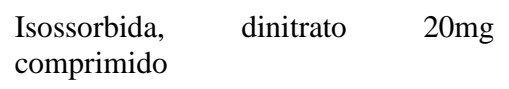 & 100.000 & 100.000 & 100.000 \\
\hline $\begin{array}{l}\text { Metoprolol, succinato de } 100 \mathrm{mg} \\
\text { comprimido }\end{array}$ & 300.000 & 300.000 & 200.00 \\
\hline $\begin{array}{l}\text { Amiodarona, Cloridrato de } 200 \mathrm{mg} \\
\text { comprimido }\end{array}$ & 500.000 & 500.000 & 500.000 \\
\hline \multicolumn{4}{|c|}{ HIPOTIREOIDISMO } \\
\hline Levotiroxina 100 mcg comprimido & 500.000 & 500.000 & 500.000 \\
\hline Levotiroxina 25 mcg comprimido & 500.000 & 500.000 & 500.000 \\
\hline Levotiroxina $50 \mathrm{mcg}$ em blister. & 500.000 & 500.000 & 500.000 \\
\hline \multicolumn{4}{|c|}{ ANTIPARKINSONIANOS } \\
\hline Levodopa + benzerazida $100+25 \mathrm{mg}$ & 100.000 & 200.000 & 200.000 \\
\hline
\end{tabular}




\begin{tabular}{|c|c|c|c|}
\hline Levodopa + benzerazida $200+50 \mathrm{mg}$ & 100.000 & 100.000 & 100.000 \\
\hline \multicolumn{4}{|c|}{ ANALGÉSICOS } \\
\hline Dipirona $500 \mathrm{mg}$ comprimido & 1.000 .000 & 1.000 .000 & 1.000 .000 \\
\hline $\begin{array}{l}\text { Dipirona } 500 \mathrm{mg} / \mathrm{ml} \text { solução oral } \\
\text { frasco } 20 \mathrm{~mL}\end{array}$ & 40.000 & 40.000 & 40.000 \\
\hline $\begin{array}{l}\text { Ibuprofeno } 50 \mathrm{mg} / \mathrm{ml} \text { suspensão oral } \\
\text { frasco } 30 \mathrm{~mL} \text {. }\end{array}$ & 30.000 & 30.000 & 30.000 \\
\hline Ibuprofeno 600 .mg comprimido & 1.000 .000 & 1.000 .000 & 1.000 .000 \\
\hline $\begin{array}{l}\text { Ácido Acetil Salícilico 100mg } \\
\text { comprimido }\end{array}$ & 2.000 .00 & 2.000 .00 & 2.000 .00 \\
\hline \multicolumn{4}{|c|}{ VITAMINAS } \\
\hline Ácido Fólico 5mg comprimido & 1.000 .000 & 1.000 .000 & 800.000 \\
\hline \multicolumn{4}{|c|}{ ANTI PENICULOSE } \\
\hline Ivermectina 6 mg comprimido & 50.000 & 50.000 & 52.000 \\
\hline \multicolumn{4}{|c|}{ ANTIALÉRGICO/ANTI-HISTAMINICOS H1 } \\
\hline Loratadina $10 \mathrm{mg}$ comprimido & 300.000 & 300.000 & 300.000 \\
\hline Prometazina $25 \mathrm{mg}$ comprimido & 100.000 & 500.000 & 500.000 \\
\hline $\begin{array}{l}\text { Dimenidrinato } 100 \mathrm{mg} \quad+\text { Vit. } \quad \text { B6 } \\
\text { Comprimido }\end{array}$ & 100.000 & 100.000 & 104.000 \\
\hline
\end{tabular}

\section{CORTICÓSTEROIDES}

Dexametasona $1 \mathrm{mg} / \mathrm{g}(0,1 \%)$

Mikania Glomerata (nome popular = guaco) xarope sem açúcar solução oral frasco $100 \mathrm{~mL}$ lacrado
30.000

30.000

33.000

EXPECTORANTE

$\begin{array}{lll}30.000 & 30.000 & 30.000\end{array}$

\section{ANTIÁCIDO GÁSTRICOS}

Omeprazol 20mg comprimido

1.500 .000

1.500 .000

1.500 .000

ANTIVIRAIS/ANTI-HELMÍTICOS

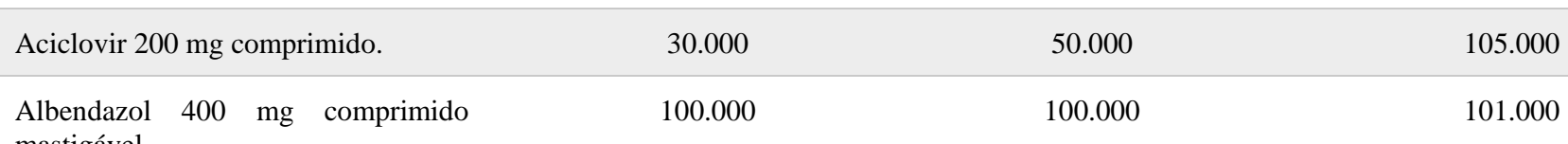

mastigável

\section{OSTEOPOROSE}

Carbonato de cálcio 500mg +

150.000

150.000

150.000

Colecalciferol 400 UI - comprimido

\section{HIPERRURICEMIAS}

Alopurinol 100 mg comprimidos

30.000

30.000

30.000

Fonte: Autores (2021). 
A Tabela 1 mostra os medicamentos que apresentaram uma maior quantidade de aquisição em 2018, 2019 e 2020 que integram o elenco da atenção básica, sendo classificados em medicamentos para hipertensão, diabetes, dislipidemias e outras doenças crônicas frequentes na população. Esses medicamentos são considerados medicamentos essenciais e indispensáveis, ocupando um papel fundamental no cuidado em saúde.

Diante disso, percebemos que os anti-hipertensivos se manteve constante, não tendo diminuição em sua quantidade licitada, além de ser a classe mais dispensada na unidade básica de saúde, isso porque a hipertensão arterial sistêmica (HAS) afeta de 11 a $20 \%$ da população adulta com mais de 20 anos, considerada a patologia que mais acarreta a população brasileira levando a umas das principais causas de morbidade, sendo considerada um problema relevante de saúde pública no Brasil e no mundo. No tratamento medicamentoso o objetivo é reduzir a morbidade e a mortalidade cardiovasculares do hipertenso, com isso tem se a necessidade de dispor desses fármacos no elenco básico de atenção para que as pessoas consigam ter o tratamento adequado e saudável (Brasil, 2001; Pellizaro \& Panckeniak, 2003).

Os medicamentos da Tabela 1 foram selecionados baseados nas prioridades de saúde e através do perfil epidemiológico do município de vitória da conquista, onde os medicamentos apresentaram segurança, eficácia terapêutica comprovada, em que são elaborados pela Comissão Municipal de Farmácia e Terapêutica (CFT) composta por uma equipe multiprofissional formada por médicos, farmacêuticos, enfermeiros e odontológico, selecionando os de melhores formas farmacêuticas para comodidade do usuário e o de menos efeitos adversos e toxicidade para poder atender as necessidades da população (Brasil, 2010).

A disponibilidades dos medicamentos e seus custos estão diretamente ligados à modalidade de aquisição adotada por cada município, portanto o processo de gestão de compras tem um grande impacto nas financias das instituições. No Brasil o processo de compra de medicamentos deve ser feito mediante pregão eletrônico. A licitação está ligada diretamente com o abastecimento de medicamentos, isto é, se alguma etapa atrasar ou falhar, todas as demais serão afetadas promovendo a escassez. (Portela, 2012; Rodrigues \& Costa, 2018).

A licitação fracassada é conceituada como a carência de licitantes interessados não atingiram as exigências de um determinado certame ou quando forem desaprovados por algum motivo. No ano de 2020 com o enfretamento da COVID-19 os desafios aumentarão junto com a falta de produtos no mercado local ou nacional em consequência das medidas preventivas que impossibilitaram a extração de matéria prima afetando a produção e importação desses produtos, reunindo a pressão do crescimento da demanda, gerando riscos de imobilização de estoques. A atividade de compras precisa lidar então com os desafios de otimizar os poucos recursos financeiros, onde houve queda nos investimentos federais nas indústrias farmacêuticas brasileiras e retração da oferta e a importância de garantia dos melhores preços numa situação de urgência, dentro do arcabouço dos requisitos da legislação brasileira. (TCU, 2018; Chaves, Serpa, Caetano, Silva, \& Luiza, 2020).

Tabela 2 - Medicamentos com quantidade licitada inferior a 30.000 unidades.

\section{FRACASSO EM LICITAÇÕES}

\begin{tabular}{cc|}
\hline Anos analisados & $\begin{array}{c}\text { Quantidade licitada em } \\
\text { porcentagem }\end{array}$ \\
\hline $\mathbf{2 0 1 8}$ & $20 \%$ \\
\hline $\mathbf{2 0 1 9}$ & $20 \%$ \\
\hline
\end{tabular}

Fonte: Autores (2021). 
Medicamentos que fracassaram nas licitações em 2020 e possuem quantitativo acima de um milhão de unidades como: Losartana $50 \mathrm{mg}$, Metformina $850 \mathrm{mg}$, Glibenclamida $5 \mathrm{mg}$, Sulfato ferroso $40 \mathrm{mg}$, paracetamol $500 \mathrm{mg}$, Carbamazepina $200 \mathrm{mg}$ e hidroclorotiazida $25 \mathrm{mg}$. Posterior ao fracasso e a urgência e demanda elevada desses itens, foi pedido um parecer jurídico, para que eles fossem comprados por dispensa de licitação ou por contrapartida estadual.

Ao comparar os valores unitários dos medicamentos observou-que: O ácido fólico $5 \mathrm{mg}$, Atenolol $50 \mathrm{mg}$, Enalapril $20 \mathrm{mg}$ e 5 mg e o Fenobarbital $100 \mathrm{mg}$ mantiveram seus valores em todos os anos, ou seja, não variaram os preços. Apenas o ácido fólico $5 \mathrm{mg}$ foi licitado em quantidade menor em 2020. 32 medicamentos sofreram aumento de 2019 para 2020, estão incluídos nesse conjunto, 11 medicamentos que tiveram seus valores atualizados no decorrer da compra, sofrendo reequilíbrio-econômicofinanceiro. Tanto o art. $\mathrm{n}^{\circ} 65$ da Lei 8.666/93, quanto o decreto 7.892/13 regulamenta essa atividade cujo os preços registrados nas atas podem ser revistos em decorrência da variação do mercado. Na comparação dos anos de 2019 para 2020, 20 medicamentos sofreram queda em seus valores, não estando relacionado ao quantitativo licitado, que foi mantido pela maioria dentre os 3 anos analisados. Ao realizar o comparativo entre 2018 e 2019, 6 medicamentos mantiveram os preços nesses anos, nesse grupo. 9 medicamentos mantiveram - se estáveis de 2019 para 2020, sendo que 5 aumentaram os preços em relação a 2018 e 4 reduziram os valores (Brasil, 1993; Brasil, 2013).

Na realidade apresentada no município de Vitória da Conquista, em 2020, segundo a Tabela 3, 11 medicamentos acima de 30.000 itens sofreram reequilíbrio-econômico-financeiro. Medicamentos como: AAS $100 \mathrm{mg}$ e Propranolol $40 \mathrm{mg}$, fármacos de uso contínuo e que são direcionados a atenção básica tiveram aumentos acima de 70\%, isso tem relação com a alta demanda dessas classes farmacológicas, visto que são utilizadas para tratamento de hipertensão arterial sistêmica (HAS), e prevenção de doenças cardiovasculares. (Pellizzaro \& Panckeniak, 2003).

Tabela 3 - Medicamentos que sofreram reequilíbrio financeiro.

\begin{tabular}{|c|c|c|c|}
\hline MEDICAMENTOS & VALOR UNITÁRIO EM 2020 & REEQUILÍBRIO & AUMENTO \\
\hline Espironolactona $25 \mathrm{mg}$ & $\mathrm{R} \$ 0,13$ & $\mathrm{R} \$ 0,15$ & $15,38 \%$ \\
\hline Digoxina 0,25 mg & $\mathrm{R} \$ 0,08$ & $\mathrm{R} \$ 0,10$ & $19,05 \%$ \\
\hline AAS $100 \mathrm{mg}$ & $\mathrm{R} \$ 0,03$ & $\mathrm{R} \$ 0,05$ & $72,41 \%$ \\
\hline Fluconazol $150 \mathrm{mg}$ & $\mathrm{R} \$ 0,35$ & $\mathrm{R} \$ 0,45$ & $28,57 \%$ \\
\hline Miconazol loção & $\mathrm{R} \$ 3,26$ & $\mathrm{R} \$ 4,30$ & $31,82 \%$ \\
\hline Propranolol $40 \mathrm{mg}$ & $\mathrm{R} \$ 0,03$ & $\mathrm{R} \$ 0,05$ & $72,41 \%$ \\
\hline Sulfa $400 \mathrm{mg}$ + trimetoprima $80 \mathrm{mg}$ & $\mathrm{R} \$ 0,12$ & $\mathrm{R} \$ 0,15$ & $27,12 \%$ \\
\hline Anlodipino 5mg & $\mathrm{R} \$ 0,03$ & $\mathrm{R} \$ 0,04$ & $32,00 \%$ \\
\hline Complexo B & $\mathrm{R} \$ 0,03$ & $\mathrm{R} \$ 0,04$ & $42,67 \%$ \\
\hline A. valproico $500 \mathrm{mg}$ & $\mathrm{R} \$ 0,37$ & $\mathrm{R} \$ 0,48$ & $29,73 \%$ \\
\hline Biperideno 2 mg & $\mathrm{R} \$ 0,17$ & 0,19 & $11,76 \%$ \\
\hline
\end{tabular}

Fonte: Autores (2021).

Quatro medicamentos do Componente Básico da Assistência Farmacêutica utilizados no âmbito da saúde mental sofreram reequilíbrio. Ácido Valpróico $500 \mathrm{mg}$ e Biperideno $2 \mathrm{mg}$ foram licitados acima de 30.000 unidades e estão expostos 
na Tabela 3. Embora medicamentos como: Fenobarbital $40 \mathrm{mg} / \mathrm{ml}$, e Haloperidol 2mg/ml não estão demonstrados nessa Tabela devido ao seu quantitativo inferior, eles também sofreram aumento significativo em seus valores, respectivamente $38,71 \%$ e $7,46 \%$.

Esses aumentos interligam-se a demanda e a questões de impacto da pandemia na sociedade. De acordo com a Organização Mundial da Saúde (OMS), o Brasil é o país que apresenta maior prevalência de depressão na América Latina. Devido a solidão enfrentada pela pandemia, existe um cenário que afeta a saúde mental da população no país, para suprir a elevada procura relacionada ao uso de fármacos nessa área, surge a Portaria $\mathrm{n}^{\circ} 2.516$, de 21 de setembro de 2020 , com intuito de autorizar excepcionalmente no momento da pandemia, a transferência de recursos financeiros de custeio para a aquisição de medicamentos do Componente Básico no âmbito da saúde mental, em virtude dos impactos sociais ocasionados pela pandemia da COVID-19. Os valores são repassados, em parcela única, com base no Índice de Desenvolvimento Humano Municipal (IDHM). Vitória da conquista se enquadra no IDHM médio, recebendo 3,09R \$ a cada habitante, totalizando R \$ 1.054.085,52 reais direcionado a compra de medicamentos para saúde mental (Brasil, 2020; Brasil, 2007).

\section{Conclusão}

Concluímos que a pandemia do COVID-19 impactou negativamente na aquisição de medicamentos do município de vitória da conquista, visto que a insuficiência de itens no mercado, seguido da demanda elevada e de preços abusivos, foram os principais motivos para o desabastecimento de medicamentos.

Houveram diferentes números de licitação que tiveram sucesso nos anos de 2018, 2019, 2020, mesmo com licitações acima de um milhão alguns medicamentos tiveram suas licitações fracassadas devido aos ajustes de preços, a urgência e a alta demanda dos itens causando uma escassez por meses de medicamentos indispensáveis para o bem-estar dos pacientes. Cerca de 11 medicamentos acima de 30.000 itens sofreram reequilíbrio-econômico-financeiro. Toda via, os anti-hipertensivos mantiveram-se sem sofrer grandes impactos nas licitações conseguindo comprar a mesma quantidade que os anos posteriores mesmo sofrendo aumento unitário. Algumas licitações sofreram fracasso por não terem licitantes interessados, tendo em vista que as indústrias não estavam conseguindo fabricar seus respectivos produtos por falta de importação de matéria-prima. Diante desta realidade sobre a COVID-19 recomenda-se que gestores tragam intervenções significativas que promovam uma melhor articulação frente ao mercado global, para que o país consiga suprir seus deveres constitucionais de provisão de medicamentos para os cidadãos, reduzindo a dependência estrangeira a longo prazo.

Destaca-se que a investigação sobre esse tema não se encerra na escrita do presente trabalho, podendo surgir investigações relacionadas ao impacto da pandemia e o consumo médio mensal da população sobre determinados medicamentos.

\section{Agradecimentos}

Os autores agradecem à Coordenação da assistência farmacêutica do município de Vitória da Conquista, Bahia, Brasil pelo auxílio na delimitação da metodologia. Agradecemos a todas as pessoas que direta ou indiretamente colaboraram com a execução deste trabalho.

\section{Referências}

Co Almeida, R. \& Alves, G. (2020). Impactos da crise mundial do coronavírus no processo produtivo da indústria farmacêutica brasileira e agravantes comerciais. Rev. Unievangélica, 2020.

Alves, A. C. \& Silva, T. O. (2019). Judicialização da saúde o Acesso a Medicamnetos no Sus em uma Secretaria Estadual. Rev. Baiana Saúde Pública, 43 (1),45-56. 
Beltrane, A., Pontarolli, S. R. D.; Cortez, E; Rieck, B. E. et al (2007). Assistência farmacêutica no SUS. CONASS, 1 (7), 186.

Bermudez, J. A. Z. Acesso a medicamentos: impasse entre a saúde e o comércio. Cadernos de Saúde Pública, 33 (9), $117-123$.

Brasil (1993). Lei No 8.666 1993. Regulamenta o art. 37, inciso XXI, da Constituição Federal, institui normas para licitações e contratos da Administração Pública e dá outras providências de 1993. https://www.jusbrasil.com.br/topicos/11311492/artigo-24-da-lei-n-8666-de-21-de-junho-de-1993.

Brasil (2007). Portaria $N^{o} 204$ 2007. Financiamento e a transferência dos recursos federais para as ações e os serviços de saúde de 2007. https://bvsms.saude.gov.br/bvs/saudelegis/gm/2007/prt0204_29_01_2007_comp.html.

Brasil (2013). Portaria $N^{\circ} 1.555$ 2013, Dispõe sobre as normas de financiamento e de execução farmacêutica no âmbito do SUS de 2013. https://bvsms.saude.gov.br/bvs/saudelegis/gm/2013/prt1555_30_07_2013.html.

Brasil (2017). Portaria N 2.001 2017. Altera a Portaria n $1.555 / G M / M S$, de 30 de julho de 2013, que dispõe sobre as normas de financiamento e execução do Componente Básico da Assistência Farmacêutica no âmbito do Sistema Único de Saúde (SUS). http://bvsms.saude.gov.br/bvs/saudelegis/gm/2017/prt2001_15_08_2017.html .

Brasil (2019). Portaria N $N^{\circ}$ 3.193, de 9 de dezembro de 2019. Altera a Portaria de Consolidação n ${ }^{\circ}$ 6/GM/MS, de 28 de setembro de 2017, para dispor sobre o financiamento do Componente Básico da Assistência Farmacêutica no âmbito do Sistema Único de Saúde (SUS). https://www.in.gov.br/en/web/dou//portaria-n-3.193-de-9-de-dezembro-de-2019-232399751.

Brasil (2020). Portaria N 2.516 , de 21 de setembro de 2020. Dispõe sobre a transferência de recursos financeiros de custeio para a aquisição de medicamentos do Componente Básico da Assistência Farmacêutica utilizados no âmbito da saúde mental em virtude dos impactos sociais ocasionados pela pandemia da COVID-19. https://brasilsus.com.br/wp-content/uploads/2020/09/portaria2516.pdf.

Brasil (2021). Controladoria geral da união. https://www.portaltransparencia.gov.br/localidades/2933307-vitoria-da-conquista.

Cerqueira, C. M. (2018.) Proposta de critérios de equidade a serem adotados no financiamento do componente básico da assistência farmacêutica considerando o princípio da universalidade. https://pesquisa.bvsalud.org/portal/resource/pt/biblio-970667.

Cerqueira, C. M. Proposta de critérios de equidade a serem adotados no financiamento do componente básico da assistência farmacêutica considerando o princípio da universalidade. http://files.bvs.br/upload/bvsecos/tcc_Cezar_Maraes_Cerqueira.pdf.

Chaves, L. A.; Serpa, G. C.; Caetano, C. M.; Silva, A. R. \& Luiza, L. V. (2020). Desabastecimento: uma questão de saúde pública global. Sobram problemas, faltam medicamentos. https://portal.fiocruz.br/documento/nota-tecnica-desabastecimento-uma-questao-de-saude-publica-global-sobram-problemas-faltam.

Costa, J. R (2019). O regime Civil-Militar no Brasil (1964-1985): Legitimações, Consenso e Colaborações. https://www.snh2019.anpuh.org/resources/anais/8/1547943350_ARQUIVO_artigo-ANPUH2019.pdf.

Cruz, A. \& Pinheiro, V. (2017). A assistência farmacêutica na rede de atenção à saúde. COSEMS.

Farinha, H. \& RIJO, J. (2020). Os Farmacêuticos Hospitalares Durante a Pandemia COVID-19. Rev Port Farmacoter, 9-19.

Feliciano, M.; Bezerra, B. F. A. \& Santos, E. G. (2020). Crescimento econômico e taxa de inflação: implicações na receita municipal e na despesa com saúde dos municípios de Pernambuco. https:/www.scielo.br/j/csc/a/ZTpMjqq5Dt8DDC684LxpRMm/?format=pdf\&lang=pt.

Gil, A. C. (1999). Métodos e técnicas de pesquisa social. Atlas.

IBGE (2021). Cidade e Estados. https://www.ibge.gov.br/cidades-e-estados/ba/vitoria-da-conquista.html.

Junqueira, D. (2020). Coronavírus expõe fragilidade das farmacêuticas, que recebem menor investimento da década sob Bolsonaro. rbrasil.org.br/2020/04/coronavirus-expoe-fragilidade-das-farmaceuticas-que-receberam-menor-investimento-da-decada-sob-bolsonaro/.

Lobô, L. S. (2019). As inovações do Pregão Eletrônico com o Decreto $\mathrm{n}^{\circ} \quad 10.024$, de 20 de setembro de 2019. ww.nucleodoconhecimento.com.br/administracao/pregaoeletronico\#: :text=De\%20acordo\%20com\%20o\%20Ministério,das\%20transferências\%20voluntárias $\% 20 \mathrm{da} \% 20$ União.

Menezes, R. A.; Moretti, B. \& Reis, A. C. (2019). O futuro do SUS: impactos das reformas neoliberais na saúde pública - austeridade versus universalidade. format=pdf\&lang=ptwww.scielo.br/j/sdeb/a/JLN5qfhCmLh4ZwY4sm4KWPt/?

Ministério da Saúde (2020). Plano de Contingência Nacional para Infecção Humana pelo novo Coronavírus COVID-19. https://portalarquivos2.saude.gov.br/images/pdf/2020/fevereiro/13/plano-contingencia-coronavirus-COVID19.pdf

Pellizzaro, M. C. \&Panckeniak, E. F. R. (2003). Assitência Farmacêutica no tratamento de doenças cardiovascular e hipertensão. Rev. Infarma, 15, (9-10), 6971.

RENAME (2001). Gerência de Assistência Farmacêutica. https://portalarquivos2.saude.gov.br/images/pdf/2014/setembro/29/Rename-2000.pdf.

Rodrigues, P. H. A.;Costa, R. D. F. \& Kiss, C. (2021). A evolução recente da indústria farmacêutica brasileira nos limites da subordinação econômica. Physis: Rev. de Saúde Coletiva, 28, (1), 1-22.

Souza, S. S. (2018). Influência da cobertura da atenção básica no enfrentamento da COVID-19. https://periodicos.unemat.br/index.php/jhnpeps/article/view/4994\#: :text=Conclusão\%3A\%20os\%20municípios\%20com\%20melhores,e\%20mortalidade\%20p ela\%20COVID-19. 